\title{
Research and Application on Health Risk Assessment Method of Coal Dust Occupational Exposure
}

\author{
Deyin Huang ${ }^{1}$, Minyan $\mathrm{Li}^{1}$, Feiran Chen ${ }^{2}$, Qian Zhang ${ }^{1}$ \\ ${ }^{1}$ Institute of Occupational Health, Tianjin Bohai Chemical Industry Group Co. Ltd, Tianjin 300051, \\ China \\ ${ }^{2}$ Tianjin Medical University, Tianjin 300070, China
}

\section{煤尘职业暴露风险评估方法研究与实例应用 \\ 黄德寅 ${ }^{1}$, 李敏嫣 ${ }^{1}$, 陈斐然 ${ }^{2}$, 张倩 ${ }^{1}$ \\ ${ }^{1}$ 天津渤海化工集团有限责任公司劳动卫生研究所, 天津 300051 , 中国 \\ ${ }^{2}$ 天津医科大学, 天津 300070 , 中国}

\begin{abstract}
Objective To research the risk assessment method of coal-worker's pneumoconiosis caused by coal dust exposure, predict the incidence of coal-worker's pneumoconiosis, and provide technical basis for occupational risk management. Method Coal-workers of a coal conveying workshop of a heat power station were chosen as objectives. The incidence of coal-worker's pneumoconiosis was predicted by logistic non-linear regression model using the concentrations of the respirable coal dust that the workers exposed to and their exposure durations. Furthermore, a practical table of the relationship between the incidence probability of coal-worker's pneumoconiosis and exposure situation was created by using $\mathrm{R}$ programming language. Results It was found that if these coal workers exposed to the respirable coal dust of the current concentration for 5 or 10 years, the risk of coal-worker's pneumoconiosis would be
\end{abstract}

基金项目: 天津市科技支撑计划重点项目（项 目编号: 13ZCZDSY02300) between $10^{-5}$ and $10^{-6}$, and for 20 or 30 years, it would be between $10^{-3}$ and $10^{-2}$. Assuming that they exposed to coal dust of this concentration for 30 years, the risk would exceed the acceptable risk level and measures should be taken. Conclusion In this study, a practical quantitative approach was proposed to assess the risk of coal-worker's pneumoconiosis caused by coal dust exposure. Based on the results, it showed that as the risk management target, the concentration and exposure duration should be well controlled to reduce the risk of coal-worker's pneumoconiosis. This method could be applied in evaluations of occupational disease hazard in construction projects and help to control and manage the risk of coal-worker's pneumoconiosis.

Keywords:Coal dust; High-risk dust; Pneumoconiosis; Risk assessment; Logistic regression model

摘要

目的研究煤尘等高危粉尘职业暴露所 致煤工尘肺的风险评估方法, 预测职业暴 
露所致尘肺病的风险概率, 为实施风险管 理提供技术依据。方法选取某热电站输煤 车间作为研究对象。采用 logistic 回归模型 的非线性回归分析, 通过接触呼吸性煤尘 浓度以及接尘工龄预测煤工尘肺发病概率; 采用计算机 $\mathrm{R}$ 语言进行接尘工龄、接尘浓 度与发病概率之间关系的风险表征结果查 表实用性研究。结果输煤车间作业场所操 作工职业暴露于目前呼吸性煤尘浓度 5 年和 10 年发生煤工尘肺的风险在 $10^{-5}-10^{-6}$, 暴露 20 年和 30 年发生煤工尘肺的风险在 $10^{-3}$ $10^{-2}$ 。按职业生涯 30 年工龄计, 发生煤工 尘肺的风险高于可接受风险水平, 需要采 取措施降低风险。结论本研究建立煤尘等 高危粉尘职业暴露所致尘肺病的风险评估 定量风险评价方法, 以劳动者接尘工龄、 呼吸性煤尘暴露浓度与煤工尘肺的风险概 率作为风险目标, 实用性强, 得以实现在 职业病风险管理中的实际应用, 为高危粉 尘职业暴露所致尘肺病的预防控制提供了 理论依据。

关键词: 煤尘; 高危粉尘; 尘肺病; 风险 评估; logistic 回归模型

\section{1. 引言}

尘肺病是我国法定职业病中的一种, 是由于在职业活动中劳动者长期吸入生产 性粉尘, 并在肺内沉积而引起的以肺组织 弥漫性纤维化为主的全身性疾病。目前尘 肺病仍是我国发病率最高的职业病, 每年 报告尘肺病例数约占职业病报告总例数 $80 \%$ 以上。根据国家公开发布的 2015-2016 年 全国职业病报告情况, 全国 31 个省、自治 区、直辖市和新疆生产建设兵团报告, 2015 年共报告职业病 29180 例。从病种分 布看, 职业性尘肺病最多, 共报告职业性 尘肺病新病例 26081 例, 其中约 93. 92\%的 病例为煤工尘肺和矽肺; 2016 年共报告职 业病 31789 例, 共报告职业性尘肺病新病 例 27992 例, 较 2015 年增加 1911 例。其 中, $95.49 \%$ 的病例为煤工尘肺和矽肺。因 此, 急需开展煤尘等生产性粉尘职业暴露 风险评价技术的研究与应用, 通过风险评 估制定尘肺病的最佳预防控制措施和防治 对策为生产性粉尘作业实施特殊管理提供
科技支撑, 预防和控制尘肺病的发生。本 文将结合某大型石化企业热电站输煤车间 的实例应用进行煤尘职业暴露风险评估方 法的介绍。，

\section{2. 对象与方法}

\section{1 对象}

某大型石油化工公司热电部输煤车间 输煤工主要负责燃料煤的接卸、存储、上 料, 操作工存在粉尘暴露时间长、暴露水 平高的特点, 并新发一例尘肺病病例。因 此, 选取该公司公司热电站输煤车间作为 研究对象, 对输煤车间输煤工职业暴露于 煤尘进行风险评估。

\section{2 方法}

根据目前国际上普遍采用的 EPA 健康 风险评价四步法的步骤进行煤尘职业暴露 风险评估, 即风险辨识、暴露评价、剂量 反应评价和风险表征等 ${ }^{[1-3]}$ 。煤尘职业暴露 致尘肺病的风险评估方法程序与内容如下:

(1) 风险辨识

通过对工作场所的煤尘职业暴露及潜 在风险进行辨识, 收集相关资料, 包括职 业史、暴露人群数量、性别、年龄分布、 暴露方式、暴露时间、工人防护条件等, 煤尘的游离二氧化硅含量及健康影响, 煤 尘职业暴露人群流行病学数据等。

（2）暴露评价

采用基于呼吸性煤尘浓度进行现场检 测的数据, 现场采样及实验室检验方法按 现行职业卫生相关标准进行。

(3) 剂量一反应评价

选择 logistic 模型作为煤工尘肺的剂 量-反应模型, 利用人群流行病学资料确定 模型参数, 得到用于评价呼吸性煤尘暴露 致煤工尘肺的剂量一反应模型; 采用统计学 软件 SPSS 19.0.0 的非线性回归拟合呼吸 性煤尘职业暴露导致煤工尘肺的剂量-反应 模型参数。

（4）风险表征

将经统计处理后的现场呼吸性煤尘检 测浓度代入剂量一反应模型直接计算, 求解 不同工龄发生煤工尘肺的风险, 对煤尘暴 
露人群在此环境下长期工作所导致煤工尘 肺的风险进行表征; 采用计算机 $\mathrm{R}$ 语言, 给出呼吸性煤尘浓度、接尘工龄与煤工尘 肺发病率的关系表。技术路线如下图所示。

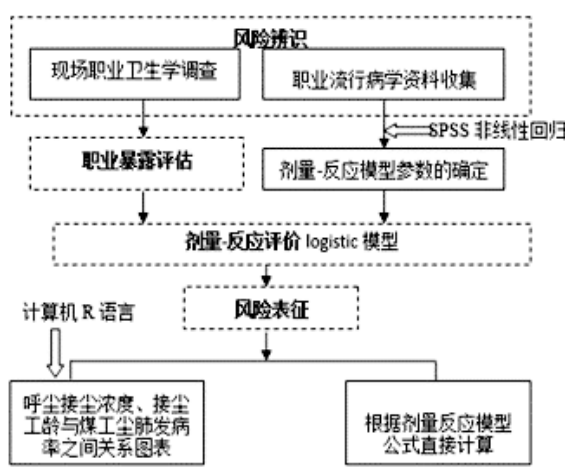

图 1 热电厂输煤工职业病风险评估技术路线

\section{3. 结果}

\section{1 输煤车间职业卫生调查}

输煤车间工艺操作主要包括火车卸煤、 煤场上煤、皮带输煤等。储存工艺: 火车 运送煤一螺旋卸煤机一煤场。输送工艺: 环式给煤机一振动给料机一皮带一篎分破 碎一皮带一煤仓。输煤车间设有全面通风 设施, 设置除尘设施共有 93 台设备, 为作 业工人配备防尘口罩等。

\section{2 工作场所煤尘暴露水平测定}

依据 GBZ159《工作场所空气中有害物 质监测的采样规范》、GBZ/T192.1《工作场 所空气中粉尘测定第 1 部分: 总粉尘浓度》、 GBZ/T192.2 《工作场所空气中粉尘测定 第 2 部分: 呼吸性粉尘浓度》进行现场采样及 实验室检测。根据职业卫生调查获取的暴 露资料, 基于现场环境浓度监测数据, 计 算呼吸性煤尘的 8 小时时间加权平均浓度, 用于煤尘所致尘肺病的风险评价。煤尘浓 度检测结果见表 1 。
表 1 工作场所空气中煤尘检测结果

\begin{tabular}{c|c|c}
\hline 岗位 & 定点采样地点 & $\begin{array}{c}\text { 检测结果 TWA } \\
\left(\mathrm{mg} / \mathrm{m}^{3}\right)\end{array}$ \\
\hline 煤管百巡检工 & - & 0.9 \\
\hline $\begin{array}{c}\text { 2\#和 3\#皮带 } \\
\text { 巡检工 }\end{array}$ & $\begin{array}{c}\text { 一电站 3\#皮带 } \\
\text { 尾部 }\end{array}$ & 2.7 \\
\hline $4 \#$ 皮带巡检工 & $\begin{array}{c}\text { 一电站 4\#皮带 } \\
\text { 尾部 }\end{array}$ & 4.5 \\
\hline
\end{tabular}

\section{3 基于 logistic 模型煤工尘肺剂量一反应} 关系

(1) Logistic 回归模型

本文选择 logistic 模型作为煤工尘肺 的剂量一反应模型。Logistic 回归模型作为 一种统计方法, 可以客观真实的呈现危险 因素与患病风险的内在联系, 并将该关系 数量化。

logistic 模型基本原理: 采用一系列 案例数据对 logistic 回归模型进行拟合; 再则, 采用该模型呈现某些自变量和因变 量取特定值之概率的关系。与此同时, 控 制特定自变量之外的一切自变量, 随之反 映该特定自变量对因变量取某特定值之概 率的决定性作用的大小。将影响煤尘职业 暴露发病的危害因素作为自变量, 煤工尘 肺患病率作为因变量, 进行多因素 logistic 回归分析, 拟合 logistic 回归模 型。下式表示某病发病概率 P 与年日接尘 浓度 $\mathrm{a}$ 、接尘工龄 $\mathrm{b}$ 之间的关系:

$$
P=\frac{\exp \left[\beta_{i 0}+\beta_{i 1} \ln (a b)\right]}{1+\exp \left[\beta_{i 0}+\beta_{i 1} \ln (a b)\right]}
$$

式中:

$\mathrm{P}$ 为不同接尘浓度、接尘工龄的煤工尘 肺发病率;

$\beta_{\text {i0 }}$ 为 logistic 回归分析的常数项;

$\beta_{i 1}$ 为 logistic 回归分析的回归系数;

a 为接尘浓度 $\left(\mathrm{mg} / \mathrm{m}^{3}\right)$;

$\mathrm{b}$ 为接尘工龄 (年)

(2) logistic 模型的参数拟合

收集公开发表国内典型行业煤工尘肺 流行病学调查资料, 根据各调查实例中的 煤尘职业暴露水平和煤工尘肺发病率数据, 拟合 logistic 模型中的参数值。本研究主 要收集并采用了以下煤工尘肺的流行病学 调查资料 ${ }^{[4-9]}$, 见表 2 。 
表 2 煤工尘肺人群流行病学调查结果

\begin{tabular}{|c|c|c|c|c|}
\hline 来源 & 行业 & 工种 & $\begin{array}{l}\text { 累积接尘量 (呼吸性煤 } \\
\text { 尘) }\left(\mathrm{mg} / \mathrm{m}^{3} \text { - 年) }\right.\end{array}$ & 累计发病率 \\
\hline \multirow{20}{*}{$\begin{array}{c}\text { 河北某煤 } \\
\text { 矿 }\end{array}$} & \multirow{20}{*}{ 煤矿 } & \multirow{20}{*}{ 采煤工 } & 8.73 & 0 \\
\hline & & & 26.18 & 0 \\
\hline & & & 43.63 & 0.0008 \\
\hline & & & 61.08 & 0.002 \\
\hline & & & 78.53 & 0.0036 \\
\hline & & & 95.99 & 0.0125 \\
\hline & & & 113.44 & 0.0156 \\
\hline & & & 130.89 & 0.0207 \\
\hline & & & 148.34 & 0.031 \\
\hline & & & 165.79 & 0.0431 \\
\hline & & & 183.25 & 0.0573 \\
\hline & & & 200.70 & 0.0787 \\
\hline & & & 218.15 & 0.0974 \\
\hline & & & 235.60 & 0.1434 \\
\hline & & & 253.05 & 0.1918 \\
\hline & & & 270.51 & 0.2402 \\
\hline & & & 287.96 & 0.288 \\
\hline & & & 305.41 & 0.3165 \\
\hline & & & 322.86 & 0.3592 \\
\hline & & & 340.31 & 0.3592 \\
\hline \multirow{2}{*}{ 内蒙古 } & \multirow{2}{*}{ 煤矿 } & \multirow{2}{*}{$\begin{array}{l}\text { 设备、皮 } \\
\text { 带司机等 }\end{array}$} & 61.08 & 0.0194 \\
\hline & & & 78.53 & 0.0441 \\
\hline $\begin{array}{l}\text { 长江 } \\
\text { 北岸 }\end{array}$ & $\begin{array}{l}\text { 港口 } \\
\text { 运输 } \\
\end{array}$ & 装卸工 & 52.36 & 0.1781 \\
\hline \multirow{2}{*}{ 山西 } & \multirow{2}{*}{ 煤矿 } & \multirow{2}{*}{ 煤 矿工 } & 16.29 & 0.01 \\
\hline & & & 13.04 & 0.005 \\
\hline
\end{tabular}

根据表 2 中呼吸性煤尘职业暴露累积 接尘量与煤工尘肺发病率间的剂量-反应关 系, 利用 SPSS 19.0.0 的 logistic 非线性 回归对收集的煤工尘肺流行病学调查数据 进行拟合。得到 logistic 模型中的参数值 $\beta_{i 0}=-20.890 、 \beta_{i 1}=3.508, R^{2}=0.898$ 。通过 检验, 可以认为接尘浓度与接尘工龄的乘 积, 即累积接尘量与发病率相关。则煤工 尘肺的剂量-反应模型为:

$$
P=\frac{\exp [-20.890+3.508 \ln (a b)]}{1+\exp [-20.890+3.508 \ln (a b)]}
$$

式中:

$a$ 为呼吸性煤尘的接尘浓度, 即 $8 \mathrm{~h}$ 时间加 权平均浓度 $\left(\mathrm{mg} / \mathrm{m}^{3}\right)$;

$b$ 为接尘工龄 (年)。

\section{4 风险表征结果}

\section{(1) 直接计算}

煤工尘肺风险计算即采用剂量-反应评 价中建立的 logistic 回归模型, 将工人实 际暴露水平数据代入上述建立的剂量-反应 模型中, 计算呼吸性煤尘暴露人群发生煤 工尘肺的风险值。各岗位煤尘接触情况及 预测的煤工尘肺风险见表 3 。

（2）基于计算机 $\mathrm{R}$ 语言的接尘浓度、接尘 工龄与发病率关系表

为了提高本研究风险评估方法的实用 性, 方便计算呼吸性煤尘职业暴露所致尘 肺病的风险, 利用 $\mathrm{R}$ 语言给出 8 小时时间 加权平均接尘浓度、接尘工龄与煤工尘肺 发病率之间关系的速查表, 即不同的接尘 浓度和 5 年、 10 年、 20 年和 30 年接尘工 
龄对应的煤工尘肺发病率可以通过查表查 得。以输煤工 20 年接尘工龄为例, 暴露于
不同 TWA 呼吸性煤尘接尘浓度下煤工尘肺 发病风险预测见表 4 。

表 3 各岗位煤尘接触情况及煤工尘肺风险

\begin{tabular}{c|c|c|c|c|c}
\hline \multirow{2}{*}{ 岗位 } & \begin{tabular}{c} 
呼尘浓度 \\
$(\mathrm{TWA})$ \\
\cline { 3 - 6 }
\end{tabular} & \multicolumn{4}{|c}{ 不同接尘工龄发生煤工尘肺的风险 } \\
\cline { 3 - 6 } & $\mathrm{mg} / \mathrm{m}^{3}$ & 5 年 & 10 年 & 20 年 & 30 年 \\
\hline 煤管百巡检工 & 0.9 & $1.66 \times 10^{-7}$ & $1.88 \times 10^{-6}$ & $2.14 \times 10^{-5}$ & $8.89 \times 10^{-5}$ \\
\hline 2\#和 3\#皮带巡检工 & 2.7 & $7.81 \times 10^{-6}$ & $8.89 \times 10^{-5}$ & $1.01 \times 10^{-3}$ & $4.18 \times 10^{-3}$ \\
\hline 4\#皮带巡检工 & 4.5 & $4.69 \times 10^{-5}$ & $5.33 \times 10^{-4}$ & $6.03 \times 10^{-3}$ & $2.45 \times 10^{-2}$ \\
\hline
\end{tabular}

表 420 年接尘工龄暴露于不同 TWA 呼吸性煤尘接尘浓度下煤工尘肺发病风险预测

\begin{tabular}{|c|c|c|c|c|c|c|c|c|c|}
\hline $\begin{array}{l}\text { 接在度 } \\
\left(\mathrm{mg} / \mathrm{m}^{3}\right)\end{array}$ & 风脸值 & $\begin{array}{l}\text { 接生度 } \\
\left(\mathrm{mg} / \mathrm{m}^{3}\right)\end{array}$ & 风脸值 & $\begin{array}{l}\text { 接生就 } \\
\left(\mathrm{mg} / \mathrm{m}^{3}\right)\end{array}$ & 风脸值 & $\begin{array}{l}\text { 接里嫬 } \\
\left(\mathrm{mg} / \mathrm{m}^{3}\right)\end{array}$ & 风脸 & $\begin{array}{l}\text { 接妇嫬 } \\
\left(\mathrm{mg} / \mathrm{m}^{3}\right)\end{array}$ & 风脸值 \\
\hline 0.5 & $2.73 \times 10^{-6}$ & 10.5 & $1.06 \times 10^{-1}$ & 20.5 & $5.53 \times 10^{-1}$ & 30.5 & $8.33 \times 10^{-1}$ & 40.5 & $9.31 \times 10^{-1}$ \\
\hline 1 & $3.10 \times 10^{-5}$ & 11 & $1.22 \times 10^{-1}$ & 21 & $5.74 \times 10^{-1}$ & 31 & $8.41 \times 10^{-1}$ & 41 & $9.34 \times 10^{-1}$ \\
\hline 1.5 & $1.29 \times 10^{-4}$ & 11.5 & $1.40 \times 10^{-1}$ & 21.5 & $5.94 \times 10^{-1}$ & 31.5 & $8.48 \times 10^{-1}$ & 41.5 & $9.36 \times 10^{-1}$ \\
\hline 2 & $3.53 \times 10^{-4}$ & 12 & $1.59 \times 10^{-1}$ & 22 & $6.14 \times 10^{-1}$ & 32 & $8.55 \times 10^{-1}$ & 42 & $9.39 \times 10^{-1}$ \\
\hline 2.5 & $7.71 \times 10^{-4}$ & 12.5 & $1.79 \times 10^{-1}$ & 22.5 & $6.32 \times 10^{-1}$ & 32.5 & $8.62 \times 10^{-1}$ & 42.5 & $9.41 \times 10^{-1}$ \\
\hline 3 & $1.46 \times 10^{-3}$ & 13 & $2.01 \times 10^{-1}$ & 23 & $6.50 \times 10^{-1}$ & 33 & $8.68 \times 10^{-1}$ & 43 & $9.43 \times 10^{-1}$ \\
\hline 3.5 & $2.51 \times 10^{-3}$ & 13.5 & $2.23 \times 10^{-1}$ & 23.5 & $6.67 \times 10^{-1}$ & 33.5 & $8.74 \times 10^{-1}$ & 43.5 & $9.46 \times 10^{-1}$ \\
\hline 4 & $4.00 \times 10^{-3}$ & 14 & $2.45 \times 10^{-1}$ & 24 & $6.83 \times 10^{-1}$ & 34 & $8.80 \times 10^{-1}$ & 44 & $9.48 \times 10^{-1}$ \\
\hline 4.5 & $6.03 \times 10^{-3}$ & 14.5 & $2.69 \times 10^{-1}$ & 24.5 & $6.98 \times 10^{-1}$ & 34.5 & $8.85 \times 10^{-1}$ & 44.5 & $9.49 \times 10^{-1}$ \\
\hline 5 & $8.71 \times 10^{-3}$ & 15 & $2.93 \times 10^{-1}$ & 25 & $7.13 \times 10^{-1}$ & 35 & $8.90 \times 10^{-1}$ & 45 & $9.51 \times 10^{-1}$ \\
\hline 5.5 & $1.21 \times 10^{-2}$ & 15.5 & $3.17 \times 10^{-1}$ & 25.5 & $7.27 \times 10^{-1}$ & 35.5 & $8.95 \times 10^{-1}$ & 45.5 & $9.53 \times 10^{-1}$ \\
\hline 6 & $1.64 \times 10^{-2}$ & 16 & $3.42 \times 10^{-1}$ & 26 & $7.40 \times 10^{-1}$ & 36 & $8.99 \times 10^{-1}$ & 46 & $9.55 \times 10^{-1}$ \\
\hline 6.5 & $2.16 \times 10^{-2}$ & 16.5 & $3.67 \times 10^{-1}$ & 26.5 & $7.53 \times 10^{-1}$ & 36.5 & $9.04 \times 10^{-1}$ & 46.5 & $9.56 \times 10^{-1}$ \\
\hline 7 & $2.78 \times 10^{-2}$ & 17 & $3.91 \times 10^{-1}$ & 27 & $7.65 \times 10^{-1}$ & 37 & $9.08 \times 10^{-1}$ & 47 & $9.58 \times 10^{-1}$ \\
\hline 7.5 & $3.51 \times 10^{-2}$ & 17.5 & $4.16 \times 10^{-1}$ & 27.5 & $7.76 \times 10^{-1}$ & 37.5 & $9.12 \times 10^{-1}$ & 47.5 & $9.59 \times 10^{-1}$ \\
\hline 8 & $4.37 \times 10^{-2}$ & 18 & $4.40 \times 10^{-1}$ & 28 & $7.87 \times 10^{-1}$ & 38 & $9.15 \times 10^{-1}$ & 48 & $9.61 \times 10^{-1}$ \\
\hline 8.5 & $5.35 \times 10^{-2}$ & 18.5 & $4.64 \times 10^{-1}$ & 28.5 & $7.97 \times 10^{-1}$ & 38.5 & $9.19 \times 10^{-1}$ & 48.5 & $9.62 \times 10^{-1}$ \\
\hline 9 & $6.46 \times 10^{-2}$ & 19 & $4.87 \times 10^{-1}$ & 29 & $8.07 \times 10^{-1}$ & 39 & $9.22 \times 10^{-1}$ & 49 & $9.63 \times 10^{-1}$ \\
\hline 9.5 & $7.70 \times 10^{-2}$ & 19.5 & $5.10 \times 10^{-1}$ & 29.5 & $8.16 \times 10^{-1}$ & 39.5 & $9.25 \times 10^{-1}$ & 49.5 & $9.65 \times 10^{-1}$ \\
\hline 10 & $9.08 \times 10^{-2}$ & 20 & $5.32 \times 10^{-1}$ & 30 & $8.25 \times 10^{-1}$ & 40 & $9.28 \times 10^{-1}$ & 50 & $9.66 \times 10^{-1}$ \\
\hline
\end{tabular}

根据上述风险预测速查表, 不同接尘 工龄的煤工尘肺发病率与接尘浓度之间的 关系图。以输煤工 20 年接尘工龄为例, 见 图 2。

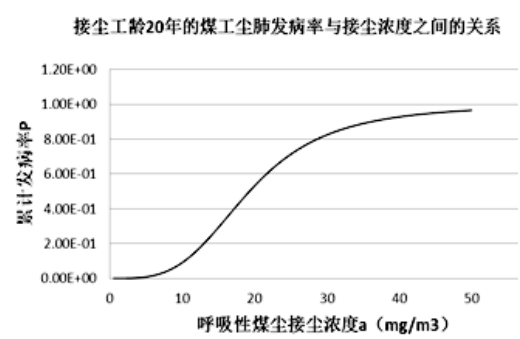

图 2 接尘工龄 20 年的煤工尘肺发病率与接
尘浓度之间的关系

\section{4. 风险控制对策建议}

由风险表征结果可以看出, 按一生 30 年工龄计, 假设工人不佩戴防尘口罩, 2 \# 和 $3 \#$ 皮带巡检工以及 4 \#皮带巡检工暴露于 煤尘的风险高于 $1 \times 10^{-3}$ 水平, 需要采取措 施降低风险。针对煤尘职业暴露存在的职 业病危害高风险, 根据风险优先权应首先 完善工程技术控制措施，主要对目前职业 病防护设施存在以下方面问题进行改造。

（1）现有岗位煤尘超标主要是皮带机落煤 时煤尘逸散, 建议考虑落煤处设置密闭形 式较好的防尘罩, 并使落煤时罩内形成微 负压, 以减少煤尘逸散。认真分析现有除 
尘系统不能有效防尘的原因, 进一步明确 除尘系统形式、位置、数量, 保证煤场及 转运、输送系统, 特别是各转运系统落煤 处煤尘浓度必须达标。

(2) 尽可能减少燃煤转运落差, 带式输送 机各转载点设的全自动消尘除尘器及喷雾 降尘设施必须保证长期使用仍能满足防尘 需要。

(3) 加强燃料车间一电站制粉系统的密闭 性, 防止煤粉的泄漏, 降低对其下层 4 \#皮 带工作环境的影响。

（4）在工艺允许的情况下, 加强湿式作业。 对煤槽螺旋卸车机增设雾化喷淋设施以及 相应的配套的水管道以及集水槽, 确保能 够实施湿式作业, 以减少扬尘。

(5) 提高除尘器吸尘罩的罩口风速, 提高 对粉尘的捕集效率, 使工作场所空气中煤 尘浓度达标。

此外, 还应采取严格的职业卫生管理 措施, 加强作业工人的个人防护, 为工人 配备防尘口罩, 并指导和监督工人正确佩 戴; 加强对作业工人进行呼吸防护用品的 使用及维护的培训和教育, 要求操作工严 格遵守操作规程; 加强防护设施的日常维 护和检修, 使其保持正常的运行状态以及 良好的通风除尘效果; 经常性地进行职业 健康检查, 并且需要对煤尘进行日常监测 等组织管理措施控制并降低风险。

\section{5. 结论与讨论}

本文建立的煤尘职业暴露风险评估方 法以常规流行病学资料为基础, 运用 logistic 回归模型的非线性回归分析, 通过 导致煤工尘肺的主要因素呼吸性煤尘接尘 浓度以及接尘工龄, 可以预测煤工尘肺发 病概率, 对输煤作业场所职业病危害进行 风险评估; 风险表征采用计算机 $\mathrm{R}$ 语言进 行接尘工龄、接尘浓度与发病概率之间关 系的可视化研究, 并进行风险表征结果速 查表实用性研究, 方便职业卫生工作者使
用查阅, 使得研究成果有很强的实用性和 可操作性。

通过实例应用证明本研究提出的煤尘 职业暴露风险评估方法可以应用于建设项 目职业病危害评价、煤尘职业暴露评估及 风险分析等工作，应用于用人单位的职业 病危害日常风险管理、煤尘等高危粉尘等 职业病高危人群管理。这对于优化劳动条 件、预防控制职业病、降低劳动者职业病 危害风险有着十分重要的意义。

\section{参考文献}

[1] Li M Y, Huang D Y, Liu M. MCSimbased occupational health risk assessment on benzene. Journal of Risk Analysis and Crisis Response, 2013, 3(3): 135-145.

[2] Zhang Q, Huang D Y, Liu M. Internal exposure simulation based on exposure related dose estimating model. Journal of Risk Analysis and Crisis Response, 2013, 3(4):175-184.

[3] Zhang Q, Huang D Y, Liu M. Study on risk evaluation based on occupational exposure evaluation and carcinogenic risk simulation. Journal of Risk Analysis and Crisis Response, 2014, 4(4): 228-232.

[4] 王永钧,张军,尹新英,等.天津市尘肺病 发生发展动态.中华劳动卫生职业病杂 志,2001,1:70-71.

[5] 华正兵,孙志谦,秦天榜.河北某煤矿工人 累积接尘量与尘肺发病危险性的剂量反应关系. 环境与职业医学, 2015,32(1):1-6.

[6] 王欣平,刘铁民. 呼吸性煤尘与尘肺的剂 量-反应关系分析.中国安全生产科学技 术, 2007,3(2):70-73.

[7] 蔡立群,韦哲.用 Logistic 回归模型研究 煤工尘肺发病规律. 中华劳动卫生职业 病杂志, 1990,8(4):223-226.

[8] 裴俊学, 李万鹏.煤尘接触量与煤工尘肺 发病关系的探讨.内蒙古煤炭经济, 2001,4:12-14.

[9] 胡建安,陈安朝,李佩珊.煤矿呼吸性粉尘 与尘肺的剂量_反应关系. 中国工业医 学杂志, 1992,5(2):66-69. 